\title{
El impacto de la era digital y su importancia en los negocios educativos en tiempos
} de Covid-19

\section{The impact of the digital era and its importance in the educational business in times of Covid-19}

DOI: $10.46932 / \mathrm{sfjdv2n5-081}$

Received in: Oct 1st, 2021

Accepted in: Dec 30th, 2021

\author{
Denisse Rosario Argüello Reyes \\ Arquitecta \\ Instituto Superior Universitario Blue Hill College \\ Av. Juan Tanca Marengo Km. 6.5 y Av. Gómez Gault, \\ E-mail: darguello@bluehill.edu.ec \\ Carla Paola Espinoza Alencastro \\ Magíster en Administración de Empresas (MBA) \\ Instituto Superior Universitario Blue Hill College \\ Av. Juan Tanca Marengo Km. 6.5 y Av. Gómez Gault, \\ E-mail: cespinoza@bluehill.edu.ec
}

\begin{abstract}
RESUMEN
Este trabajo analiza la importancia del mercado educativo y la inclusión de medios tecnológicos para el proceso de transformación de las empresas tradicionales a negocios digitales; la investigación es documental de tipo descriptiva - exploratoria; en esta se examinó documentación científica y representativa de instituciones educativas de educación superior; esencialmente sobre servicios digitales y los efectos del COVID-19 en la manera de hacer negocios.

El objetivo de este paper fue indagar el proceso transformador de los servicios tradicionales a actividades comerciales con plataformas tecnológicas en el área educativa. A modo de resultado de este análisis, se observa una correlación entre los sucesos de la pandemia y el ineludible cambio de los negocios en la educación superior; comprobándose una innovación efectiva de una instrucción flexible y herramientas especializadas para la era actual.
\end{abstract}

Palabras claves: Covid-19, medios digitales, educación superior, mercado educativo, innovación.

\begin{abstract}
This document analyzes the importance of the education market and the inclusion of technological means for the transformation process of traditional firms and digital businesses; this research is an exploratory descriptive documentary; in which scientific and representative documentation from higher education institutions have been examined; essentially about digital services and the impacts that COVID-19 has had in the way of making business.

This paper's objective was to investigate the transformational process of traditional services in the educational area to commercial activities with technological platforms. As a result of this analysis, we can observe a correlation between situations originated due to pandemics and the unavoidable change in higher education businesses; proving an effective innovation of flexible instructions and specialized tools applicable in the current era.
\end{abstract}


Keywords: Covid-19, digital media, higher education, education market, innovation

\section{INTRODUCCIÓN}

La pandemia COVID-19 ha creado disrupciones en distintos espacios del dinamismo humano. La enseñanza superior ha sido afectada debido al cierre total de las sedes formativas en gran parte del mundo. La singularidad de la educación a distancia, principalmente en soporte digital, apareció para suplir soluciones de emergencia a dicha crisis.

En ningún tiempo de la historia se ha producido una clausura mundial de las infraestructuras educativas presenciales tal como lo sucedido con la pandemia provocada por el COVID-19. Según la United Nations Educational, Scientific, and Cultural Organization. International Affairs Office (UNESCO), los gobiernos de alrededor 200 naciones declararon el cierre total o parcial de centros educativos (García, 2021). Conforme a la organización internacional, aproximadamente 1600 millones de niños, adolescentes y jóvenes se ha visto comprometida a nivel mundial por esta pandemia, $91 \%$ del total (figura 1), de la misma manera más de 60 millones de docentes dirigidos a generar una revolución educativa en el método de enseñanza tecnológica digital (IESALC-UNESCO, 2020).

Figura 1 Alumnos afectados por la pandemia COVI-19

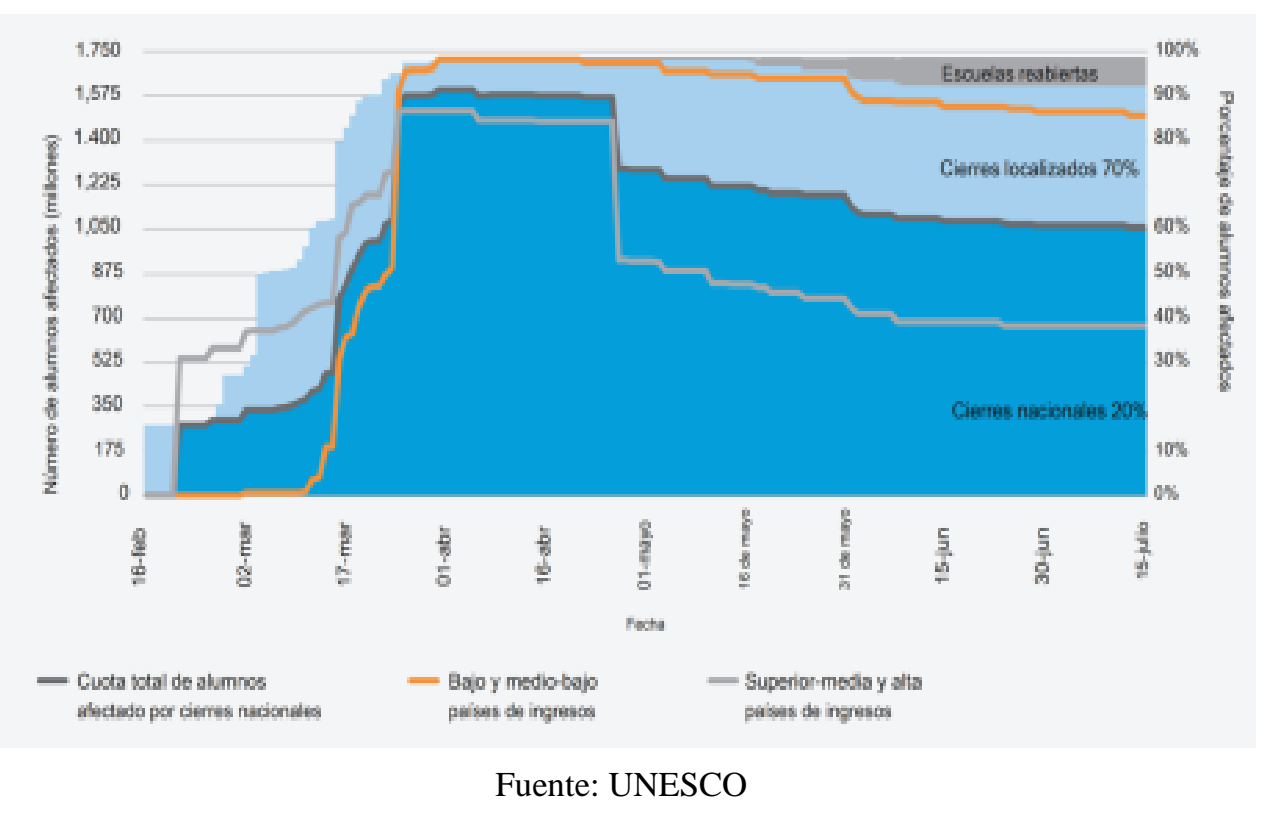

Por consiguiente, UNESCO exhortó a los regímenes, y actualmente aún la extiende, al uso de procedimientos de aprendizaje a distancia, produciendo las contingencias que hoy ofrecen las tecnologías digitales con variadas iniciativas como plataformas y aplicaciones, algunas gratuitas, que acceden el estudio con la interacción de materiales y docentes de manera online o de manera asíncrona. 


\section{DESARROLLO}

Como manifiesta Hernández (2020), la necesidad de examinar nuestro papel como agentes sociales, el de los establecimientos en su pertinencia y compromiso social, como gestores formativos qué proponen y sugieren contenciones adecuadas con el soporte de las tecnologías de información y comunicación, permitiendo potenciarlas desde casa para fomentar los conocimientos de aprendizajeenseñanza de los estudiantes y propiciar escenarios colaborativos.

La perspectiva de lo que involucra y representa la «gestión formativa» propone analizar el accionar de los gestores educativos que exhortan a lograr, desenvolver y reforzar, "un conjunto de competencias, a fin de lograr un impacto positivo con sus prácticas, ya sea como hacedores y tomadores de decisiones de políticas públicas, de los directivos en los establecimientos educativos, o en su rol como docentes (Hernández, 2020).”

Para Moncayo (2006) la gestión educativa es una “... actividad práctica, un saber hacer que incluye una pluralidad de conocimientos y actividades relacionados con aspectos esenciales del funcionamiento de las organizaciones educativas, entre otras:

- Generar, acordar y alcanzar objetivos

- Establecer funciones

- Utilizar formas adecuadas de liderazgo y dirección

- Buscar maneras adecuadas de dirigir la iniciativa institucional hacia una acción educativa y medio ambiental de calidad."

En tiempos de coronavirus la educación en línea se traduce en una solución a esta pandemia. Esta crisis nos revela que es primordial la adquisición y perfeccionamiento de competencias en TICs y también en habilidades blandas.

Desde el punto de vista de Hodges, C.B., Moore, S.L., Lockee, B.B., Trust, T., \& Bond, M.A. (2020), esta instrucción en línea efectiva es consecuencia de un diseño instruccional cuidadoso, utilizando un modelo sistemático de planificación. El proceso instruccional tiene un impacto en la calidad de la instrucción, y es este cuidadoso proceso en la enseñanza remota de emergencia. Los modelos de soporte vigentes incluyen plataformas digitales con diseño de curso completo, desarrollo de contenido, entrenamiento y asistencia técnica de la gestión de aprendizaje con especialistas de la facultad.

De acuerdo con Diario El Universo (2021), para las universidades ecuatorianas la modalidad de estudio ha sido primordialmente presencial, sin embargo, debido a la pandemia éstas han tenido que innovar en el proceso educativo de manera radical. Los centros de estudios superiores y sus educandos han encontrado variadas ventajas en este sistema. Hoy por hoy, la teleducación ha estimulado el uso de plataformas digitales tales como Zoom, Meet, Microsoft Teams, Blackboard, Moodle, AVAC. 


\section{CONCLUSIÓN}

Por definición la innovación radical conlleva un alto riesgo para las empresas que lo implementan. No obstante, adoptar esta medida podría resultar en que las organizaciones tengan una mayor ventaja competitiva. El Covid-19, presentó un escenario insólito, jamás vivido por nuestras generaciones y en esta ocasión, por lo tanto, abstenerse de aplicar la innovación radical era un factor de riesgo. Las instituciones educativas tuvieron que adaptarse creativamente ya no para generar ventaja competitiva sino para continuar existiendo.

En las universidades privadas, se migró todo el servicio educativo a plataformas virtuales impulsando su uso entre todos los miembros de la comunidad. Esta acción buscaba mantener la vigencia de las instituciones, así como también continuar con el cumplimiento de sus objetivos y funciones. En tiempos denominados ahora como "pre-pandémicos", ya se venía incorporando el uso de plataformas tecnológicas en las metodologías presenciales, pero de manera parcial, como herramientas complementarias.

De acuerdo con García (2020), en el modelo de enseñanza y aprendizaje no tradicional, donde existe la colaboración y conectividad, las metodologías con TIC, TAC y TEP tienen la ventaja de aumentar el nivel de asociación entre el docente y alumno, de manera que los estudiantes se conviertan en creadores de contenido y de vínculos entre los contenidos del curso.

El equipo docente ha manifestado que el balance ha sido positivo respecto a la captación de conocimiento de los estudiantes y al incremento del seguimiento de clases. Sin embargo, para los profesores, el desarrollo y uso de esta nueva tecnología requirió un mayor esfuerzo y dedicación en los momentos iniciales. Por lo tanto, los maestros consideraron que la herramienta tiene un gran potencial en el inicio de la educación digital, desarrollando metodologías colaborativas tipo "b-learning" en la educación superior que mejoran las metodologías de aula actuales. (Isabel Legaz, Aurelio Luna; 2021)

El servicio educativo ha logrado mantenerse vigente y activo gracias a los sistemas digitales. Se ha vuelto imperativo por consiguiente conocer y dominar las herramientas digitales pues son el puente comunicacional entre educadores y educandos. Alumnos y docentes han incorporado las plataformas como parte esencial en sus hábitos de estudio y trabajo. Este uso cotidiano ha impulsado y generado una constante actualización y evolución en las plataformas las cuales ya se han vuelto imprescindibles en el servicio educativo y buscan mantenerse de tal forma incluso cuando se vuelva a la presencialidad. Por lo expuesto previamente se puede afirmar la innegable necesidad y mutualismo entre las plataformas tecnológicas - digitales y los negocios que brindan servicios educativos por lo que sería interesante preguntarnos: ¿los negocios educativos que adoptan estas innovaciones serían sustentables a largo plazo en el mercado de la educación superior? 


\section{REFERENCIAS}

Campos-Sánchez, Shirley Tatiana et al (2021). Los negocios digitales y su importancia en la era actual. Dominio de las Ciencias, [S.1.], v. 7, n. 1, p. 1029-1043, feb. 2021. ISSN 2477-8818. Disponible en: 〈https://www.dominiodelasciencias.com/ojs/index.php/es/article/view/1755>. Fecha de acceso: 01 nov. 2021 doi:http://dx.doi.org/10.23857/dc.v7i1.1755

García, Laura (2020). Coronavirus. Educación y uso de tecnologías en días de pandemia

Ciencia UNAM. http://ciencia.unam.mx/leer/1006/educacion-y-uso-de-tecnologias-en-dias-de-pandemia García Aretio, Lorenzo (2021). COVID-19 y educación a distancia digital: preconfinamiento, confinamiento y posconfinamiento RIED. Revista Iberoamericana de Educación a Distancia, vol. 24, núm. 1,2021

Hernández Mondragón, Alma Rosa (2020). COVID-19: el efecto en la gestión educativa. RELAIS, vol. 3 , no. $1,2020$.

Hodges, C.B., Moore, S.L., Lockee, B.B., Trust, T., \& Bond, M.A. (2020). The Difference between Emergency Remote Teaching and Online Learning. Educational Review.

IESALC-UNESCO (2020). El coronavirus-19 y la educación superior: impacto y recomendaciones. Disponible en: https://www.iesalc.unesco.org/2020/04/02/el-coronavirus-covid-19-y-la-educacionsuperior-impacto-y-recomendaciones/

Informes. (2021). Universidades de Ecuador se anclan a la virtualidad de la educación, pero hay $\begin{array}{llll}\text { incertidumbre } & \text { para } & \text { este } & 2021\end{array}$ https://www.eluniverso.com/noticias/2021/01/10/nota/9343705/universidades-educacion-matriculaspandemia-covid-teleducacion/

Legaz, I. Luna, A. (2021). Implementation of digital media in higher education. An experience from the classroom. South Florida Journal of Development. Recuperado de https://southfloridapublishing.com/ojs/index.php/jdev/article/view/606

Moncayo González, L.G. (2006). Delimitando el concepto de gestión escolar. Revista de Educación educar. Núm. 39, México, octubre-diciembre. 